\title{
Imaging of endometrial osseous metaplasia-an uncommon but treatable cause of infertility
}

\author{
Abdul Haseeb Wani ${ }^{1 *}$ (D, Arshed Hussain Parry ${ }^{2}$, Imza Feroz ${ }^{3}$, Majid Jehangir ${ }^{1}$ and Masarat Rashid ${ }^{4}$
}

\begin{abstract}
Background: Endometrial osseous metaplasia (EOM) is an uncommon condition characterised by metaplastic transformation of endometrial tissue into osteoblasts (mature or immature bone in the endometrium). Etiopathogenesis of EOM is explained by multiple putative mechanisms like dystrophic calcification, metaplastic ossification, retained foetal bones after abortions and genito-urinary tuberculosis. EOM has varied clinical presentation ranging from patient being asymptomatic to secondary infertility. Although hysteroscopy is the gold standard for its diagnosis and treatment, non-invasive imaging comprising chiefly of ultrasonography (USG) is increasingly becoming the mainstay of diagnosis. We aim to present the imaging findings in EOM to acquaint radiologists and gynaecologists with this condition to avert misdiagnosis of this uncommon yet treatable cause of infertility.

Results: Mean age of patients was $31.4 \pm 5.4$ (S.D) years. USG revealed linear or tubular densely echogenic endometrium with posterior acoustic shadowing in all the 14 patients. MRI in 3 patients revealed diffuse or patchy areas of T1W and T2W hypointense signal intensity with unilateral $(n=2)$ and bilateral $(n=1)$ ovarian cysts. One patient who underwent CT scan revealed dense endometrial calcification. Histopathologic examination (HPE) revealed lamellar $(n=6)$ or trabecular $(n=4)$ bone within endometrium $(E O M)$ and inflammatory cells with calcification in four patients (calcific endometritis). Twelve patients conceived after dilatation and curettage within 15 months.
\end{abstract}

Conclusion: Familiarity with the imaging appearances of EOM is indispensable to clinch this diagnosis and avert misdiagnosis of this rare but potentially treatable cause of infertility. USG is usually sufficient for diagnosis. MRI and CT are only supplementary tools in difficult clinical scenarios.

Keywords: Endometrium, Osseous metaplasia, Ultrasonography, Endometrial calcification, Dilatation and curettage

\section{Background}

Etymologically endometrial osseous metaplasia (EOM) meaning metaplastic transformation of endometrial tissue into osteoblasts is an uncommon condition characterised by the presence of mature or immature bone in the endometrium [1]. Various theories have been propounded to explain its aetiology with dystrophic calcification, metaplastic ossification, heterotopia, retained

\footnotetext{
*Correspondence: soberseeb@gmail.com

'Department of Radiodiagnosis, Government Medical College, Srinagar, Jammu and Kashmir 190010, India

Full list of author information is available at the end of the article
}

foetal bones after spontaneous/missed/incomplete or therapeutic abortion, chronic inflammation (genito-urinary tuberculosis) and extended post-abortion estrogenic treatment being the most plausible ones [2-4]. EOM has varied clinical presentation ranging from patient being asymptomatic to secondary infertility [5-7]. Within this wide spectrum, patients can also present with menstrual irregularities (menorrhagia or oligomenorrhea) and recurrent abortions [8,9]. Although hysteroscopy is the gold standard for its diagnosis and treatment, noninvasive imaging comprising chiefly of ultrasound is increasingly becoming the mainstay of diagnosis and is

\section{Springer Open}

(c) The Author(s). 2020 Open Access This article is licensed under a Creative Commons Attribution 4.0 International License, which permits use, sharing, adaptation, distribution and reproduction in any medium or format, as long as you give appropriate credit to the original author(s) and the source, provide a link to the Creative Commons licence, and indicate if changes were made. The images or other third party material in this article are included in the article's Creative Commons licence, unless indicated otherwise in a credit line to the material. If material is not included in the article's Creative Commons licence and your intended use is not permitted by statutory regulation or exceeds the permitted use, you will need to obtain permission directly from the copyright holder. To view a copy of this licence, visit http://creativecommons.org/licenses/by/4.0/. 
gaining wider acceptance for initial diagnosis due to its easy availability, non-invasive nature and lack of ionising radiation [10]. Given the rarity of this condition there is sparse literature available on EOM particularly the imaging features. Whatever miniscule literature is present that is in the form of case reports. We aim to present the imaging findings in EOM to acquaint radiologists and gynaecologists with this condition in order to avert misdiagnosis of this uncommon yet treatable cause of infertility.

\section{Methods}

This was prospective descriptive single-centre study covering a study period of 3 and half years from April 2016 to December 2019. During the study period, a total of 411 infertile women comprising of 198 (48.2\%) patients of primary infertility and $213(51.8 \%)$ of secondary infertility attended the out-patient clinic of our hospital. Patients were considered infertile when they failed to conceive after unprotected and regular sexual intercourse for a period of 1 year. Primary infertility refers to inability to conceive even once whereas secondary infertility is defined as failure to conceive after a previous conception. Informed consent was taken from all the patients with institutional review board approval obtained for the study. Relevant clinical history, detailed general physical examination, and local examination were recorded. All the biochemical parameters were recorded including liver function tests, renal function tests, fasting blood sugar, TSH, T3, T4, FSH, LH, FSH/LH ratio, CA-125 and prolactin levels.

All the patients were subjected to preliminary transabdominal ultrasound (TAS) examination of pelvis. Fourteen patients were suspected to have EOM on the basis of TAS findings and were included in this study. These patients subsequently underwent transvaginal ultrasound (TVS) to confirm the findings of TAS. Patients with history of intra-uterine contraceptive device insertion in past were excluded from the study. TAS examination of the pelvis was performed using LOGIQ P5 (GE Healthcare, Chicago, IL, USA) ultrasound machine with convex low frequency ultrasound probe $4 \mathrm{C}(1.6$ to $4.6 \mathrm{MHz})$ in all patients. TVS examination was performed using $3 \mathrm{C} 8$

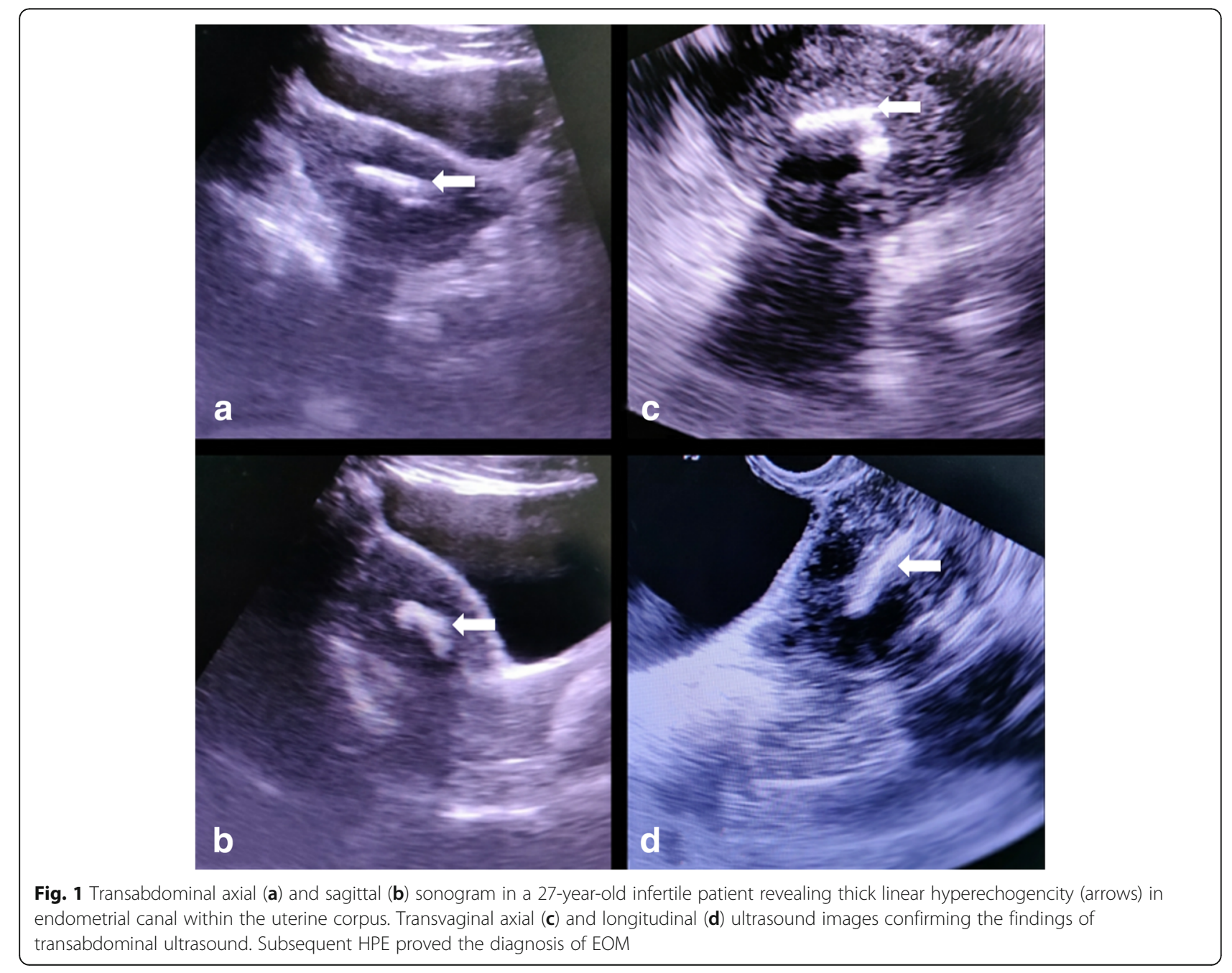




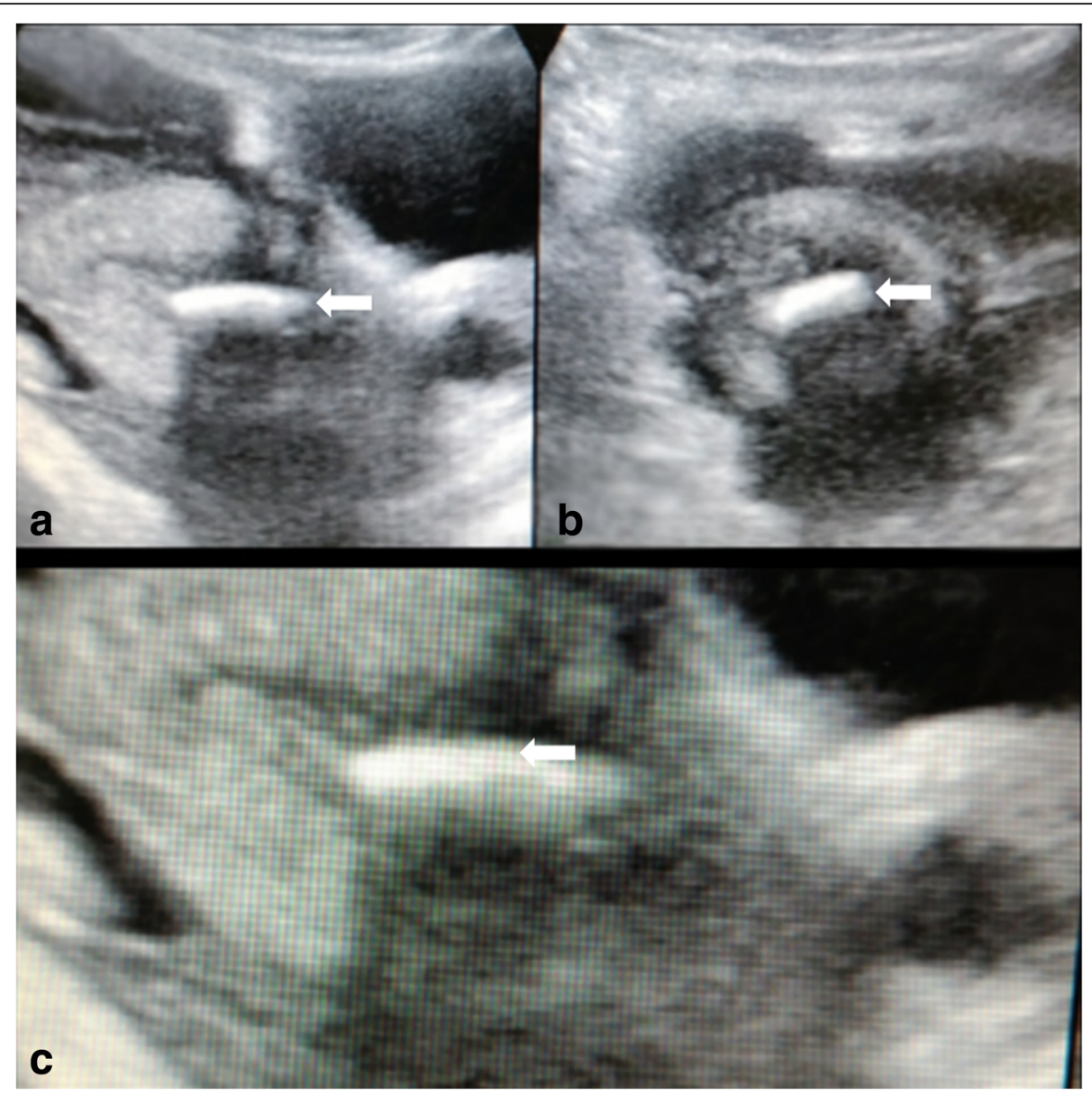

Fig. 2 Sagittal (a), axial (b) and magnified sagittal (c) transabdominal ultrasound images in a 32-year-old patient with previous history of postabortal instrumentation showing linear hyperechogencity with posterior acoustic shadowing in endometrial cavity of lower uterine corpus (arrows)

endo-cavitary probe ( 3 to $8 \mathrm{MHz}$ ). Longitudinal and transverse ultrasound images of uterus, endometrial lining and bilateral adnexae were obtained. Three patients had an additional magnetic resonance imaging (MRI) of pelvis and one patient additionally underwent computed tomographic (CT) abdomen. MRI was performed in these three patients owing to preliminary USG examination showing complex ovarian cysts in addition to USG findings of EOM. One patient who underwent CT abdomen had pulmonary tuberculosis with recurrent abdominal pain. All these 14 patients finally underwent hysteroscopic examination with dilatation and curettage. The retrieved uterine tissue was put in $10 \%$ formalin, and the vial was transported to histopathological laboratory. The tissue was kept for overnight fixation in formalin. Subsequently, the tissue was grossed and processed routinely. The slides obtained were stained with haematoxylin and eosin (H\&E) and subjected to microscopic examination under scanner, low power and high power view. . Patients were subsequently followed for an average period of 15 months (range 9 months to 24 months) to monitor the response to endometrial curettage in the form of future conception.

\section{Results}

Mean age of patients was 31.4 \pm 5.4 (S.D) years. Secondary infertility and primary infertility were the chief complaints in $12 / 14(85.7 \%)$ and $2 / 14(14.3 \%)$, respectively. Menstrual irregularities were present in $5(35.7 \%)$ patients. Pelvic pain was associated in $4(28.6 \%)$ patients. Recurrent abdominal pain was seen in 1 patient. Ten (71.4\%) patients gave a history of previous conception followed by spontaneous missed abortion at 9 to 17 weeks of gestational age followed by dilatation and curettage. However, in remaining four (28.6\%) patients, history of previous abortion or dilatation and curettage was not forthcoming. Two patients in addition to being secondarily infertile presented with vaginal discharge. History of previously diagnosed pulmonary tuberculosis was forthcoming in one patient; however, the patient had left 
anti-tubercular treatment (ATT) midway. There was no history of intra-uterine contraception device (IUCD) insertion in any patient. The overall physical examination was unremarkable and bimanual pelvic examination was normal in 12 patients. Two patients with vaginal discharge had mild cervical tenderness on per vaginal examination. The routine laboratory investigations, hormonal and biochemical profile of all patients were within normal range as follows: T4 (SIU-standard international unit) $114.04 \pm 6.36$, T3 (SIU) $2.29 \pm 0.24$, TSH (SIU) 4.44 \pm 0.73 , fasting blood sugar levels $86 \pm 9 \mathrm{mg} / \mathrm{dl}$, serum bilirubin levels $0.8 \pm 0.2 \mathrm{mg} / \mathrm{dl}$, albumin level $5.5 \pm 0.4 \mathrm{~g} / \mathrm{L}$, serum creatinine level $0.75 \pm 0.2 \mathrm{mg} / \mathrm{dl}$, blood urea nitrogen level $19.3 \pm 4.2 \mathrm{mg} / \mathrm{dl}$, FSH levels on day 3 of menstrual cycle $11.23 \pm 3.63 \mathrm{mIU} / \mathrm{ml}$, LH levels $9.1 \pm 1.2$ $\mathrm{mIU} / \mathrm{ml}, \mathrm{LH} / \mathrm{FSH}$ ratio $<1$, prolactin level $18 \pm 4 \mathrm{ng} / \mathrm{ml}$, testosterone levels $30 \pm 3 \mathrm{ng} / \mathrm{dl}$ and CA-125 levels $19 \pm 3$ $\mathrm{U} / \mathrm{ml}$. The semen analysis of the husband was normal in each case. Four patients had undergone hysterosalpingography (HSG) in previous 1 year and all four had normal peritoneal spill of contrast suggesting tubal patency.

Longitudinal and transverse ultrasound of the pelvis revealed well defined, linear or tubular echogenic area with dense posterior acoustic shadowing within the endometrial canal of uterine corpus in nine cases (Figs. 1, 2, and 3) which extended to the upper part of the cervix along the endocervical canal in the remaining five cases. The anterior and posterior myometrial walls were normal in morphology with normal echotexture in all cases. No fibroid or adenomyosis was seen. Cervix was mildly bulky in one patient with no cervical fibroid or mass. Pertinent negative USG findings were absence of hydrometra or hydrosalpinx. Eleven patients had normal bilateral ovaries with normal ovarian volume and folliculometry. Two patients had unilateral complex ovarian cysts. One patient with vaginal discharge had bulky uterus with bilateral adnexal complex cystic lesions and mild free fluid in pouch of Douglas.

On the basis of history, clinical examination and ultrasound findings, a presumptive diagnosis of endometrial osseous metaplasia (EOM) was made in all the 14 patients. Three patients with additional finding of complex adnexal cysts on USG were further examined by noncontrast MRI pelvis which revealed scattered areas of T2-weighted (T2W) hypointense signal intensity within the background hyperintense endometrium in all cases. Corresponding T1W images showed hypointense signal intensity in all the three patients (Fig. 4). Two patients showed unilateral ovarian cysts with hyperintense T1W and T2W signal intensity with non-suppression of signal on T1W fat-sat images suggesting a diagnosis of ovarian haemorrhagic cysts. One patient had bilateral complex adnexal cysts with T1W hypointense and T2W hyperintense signal intensity with free fluid in pelvis. Subsequent polymerase chain reaction (PCR) of menstrual blood detected nucleic acid of acid-fast bacilli (AFB) in this patient thus confirming genital tuberculosis. CT of abdomen, done in one patient for recurrent abdominal pain, revealed multiple calcified mesenteric and retroperitoneal nodes in addition to dense endometrial ossification with attenuation values similar to spongy bone

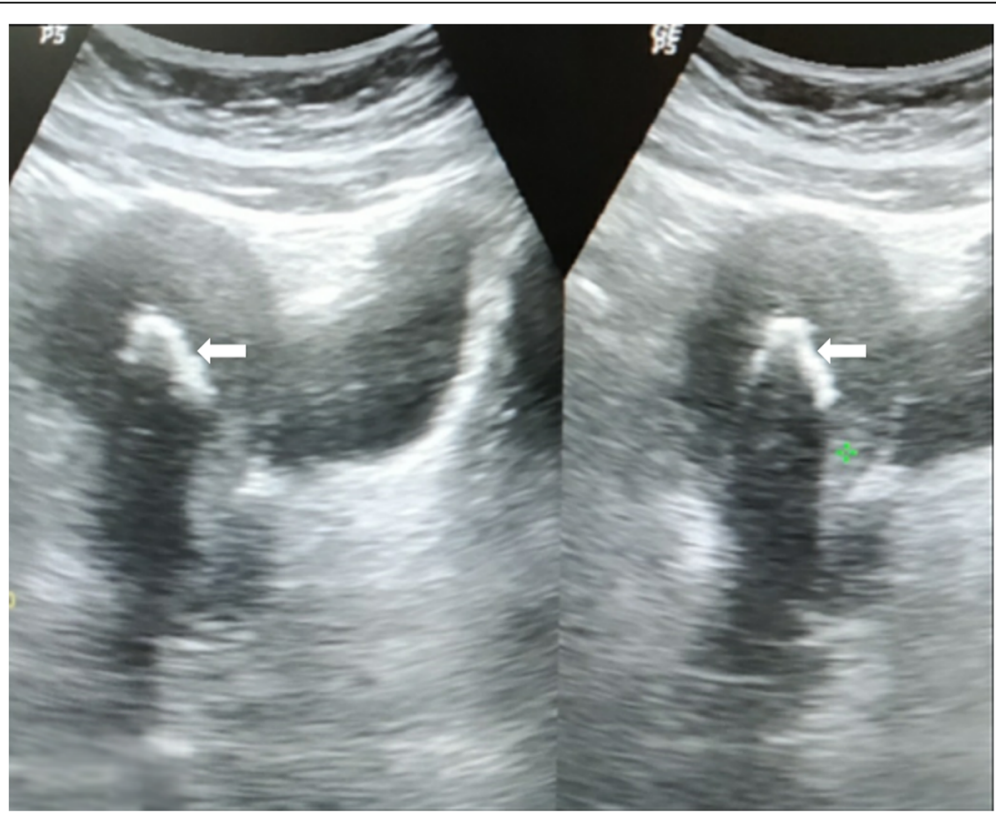

Fig. 3 Sagittal split-screen transabdominal ultrasound images in a 28-year-old infertile patient revealing curvilinear echogenic area in endometrial canal with dense posterior acoustic shadowing (arrows). Subsequent HPE revealed calcific endometritis 
(Fig. 5). With the preliminary sonographic diagnosis of EOM, all the 14 patients underwent hysteroscopy with dilatation and curettage. Hysteroscopy revealed patchy or diffuse ivory white ossified endometrium (Fig. 6) in 11 patients whereas 3 patients showed shaggy discoloured endometrium. Histopathological examination of the retrieved endometrial tissue revealed non-specific lymphocytic infiltrate with interspersed lamellar bone in six (42.8\%), trabecular bone in four (28.5\%) patients with scanty endometrial glands and stroma in various hormonal phases confirming the diagnosis of osseous metaplasia of endometrium (Figs. 7 and 8). Remaining four (28.5\%) patients showed chronic inflammatory (granulocytic) infiltrate with specks of calcification within the endometrium suggesting diagnosis of calcific endometritis. These four patients were given a diagnosis of endometrial tuberculosis and were started on ATT. On follow-up, 11 patients conceived in the subsequent 15-month follow-up period whereas 1 patient conceived after 20 months and 2 have not conceived till the end of study.

\section{Discussion}

The putative hypothesis for the genesis of endometrial osseous metaplasia is an area of active research. Various theories have been propounded which may also explain the varied names by which it has been labelled since its early description in 1884 by Virchow who attributed the formation of ossified tissue within endometrium to de novo differentiation of fibroblasts into osteoblasts [11]. Nine out of 10 (90\%) of HPE proven EOM had a history of abortion followed by dilatation and curettage in our study whereas 1 patient had no previous history of abortion or uterine instrumentation. Post-abortion instrumentation was the most common putative aetiology of EOM in our study. Chronic inflammation of endometrium due to post-abortal tissue likely stimulates release of various inflammatory mediators causing metaplastic transformation of stromal cells into osteoblasts [12]. Why this metaplastic transformation occurs in very few patients is a matter of speculation and active research. To account for this discrepancy, superoxide dismutase

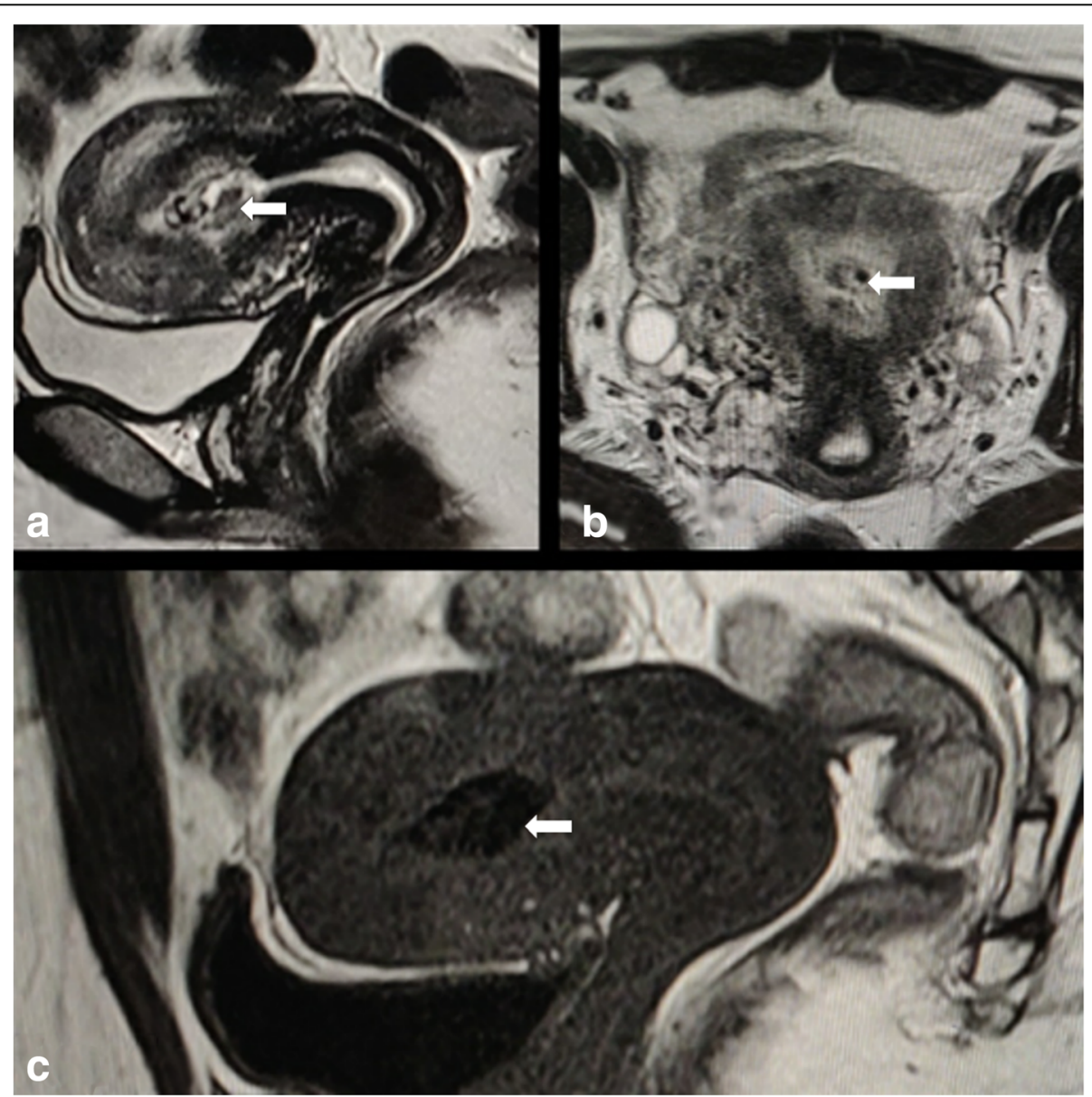

Fig. 4 Fast spin echo T2W sagittal (a) and axial (b) images of uterus in a 29-year-old infertile patient reveal patchy areas of hypointensity (arrows) within the endometrial canal in the uterine body. T1W sagittal (c) image reveals markedly hypointense and thick endometrial signal (arrows) compared to the myometrium. Subsequent HPE proved the diagnosis of EOM 


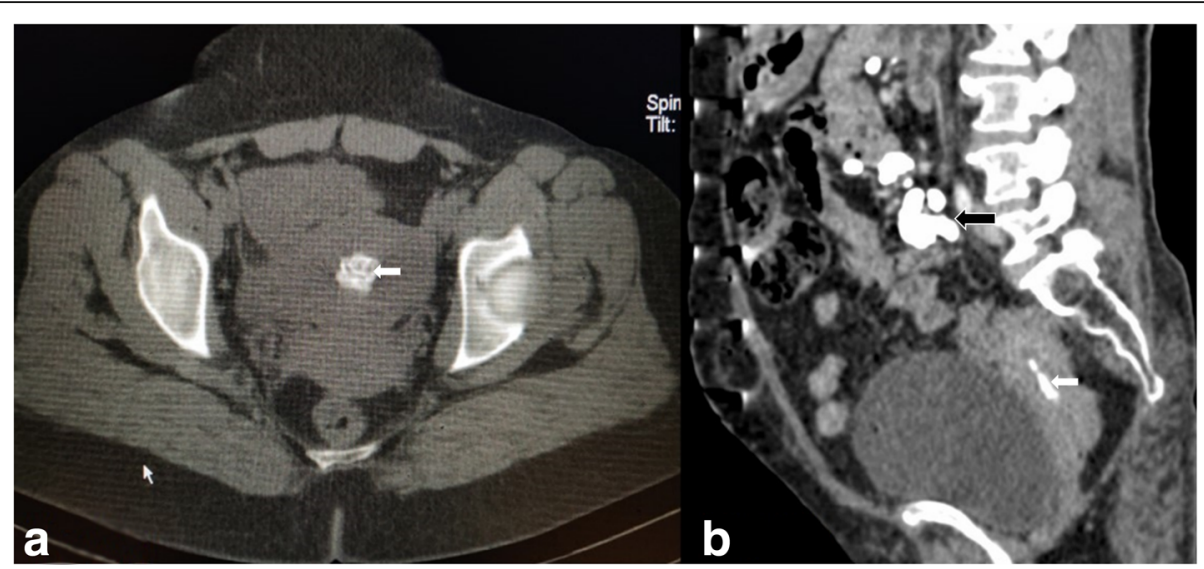

Fig. 5 Axial (a) and sagittal (b) non-contrast $\subset$ T images of pelvis and lower abdomen in a 30-year-old infertile patient with a history of old pulmonary tuberculosis and recurrent abdominal pain revealing dense endometrial calcification (white block arrows) with Hounsfield attenuation of $520 \mathrm{HU}$ similar to adjacent pelvic bone and calcified retroperitoneal nodes (black block arrow). Subsequent HPE clinched the diagnosis of calcific endometritis

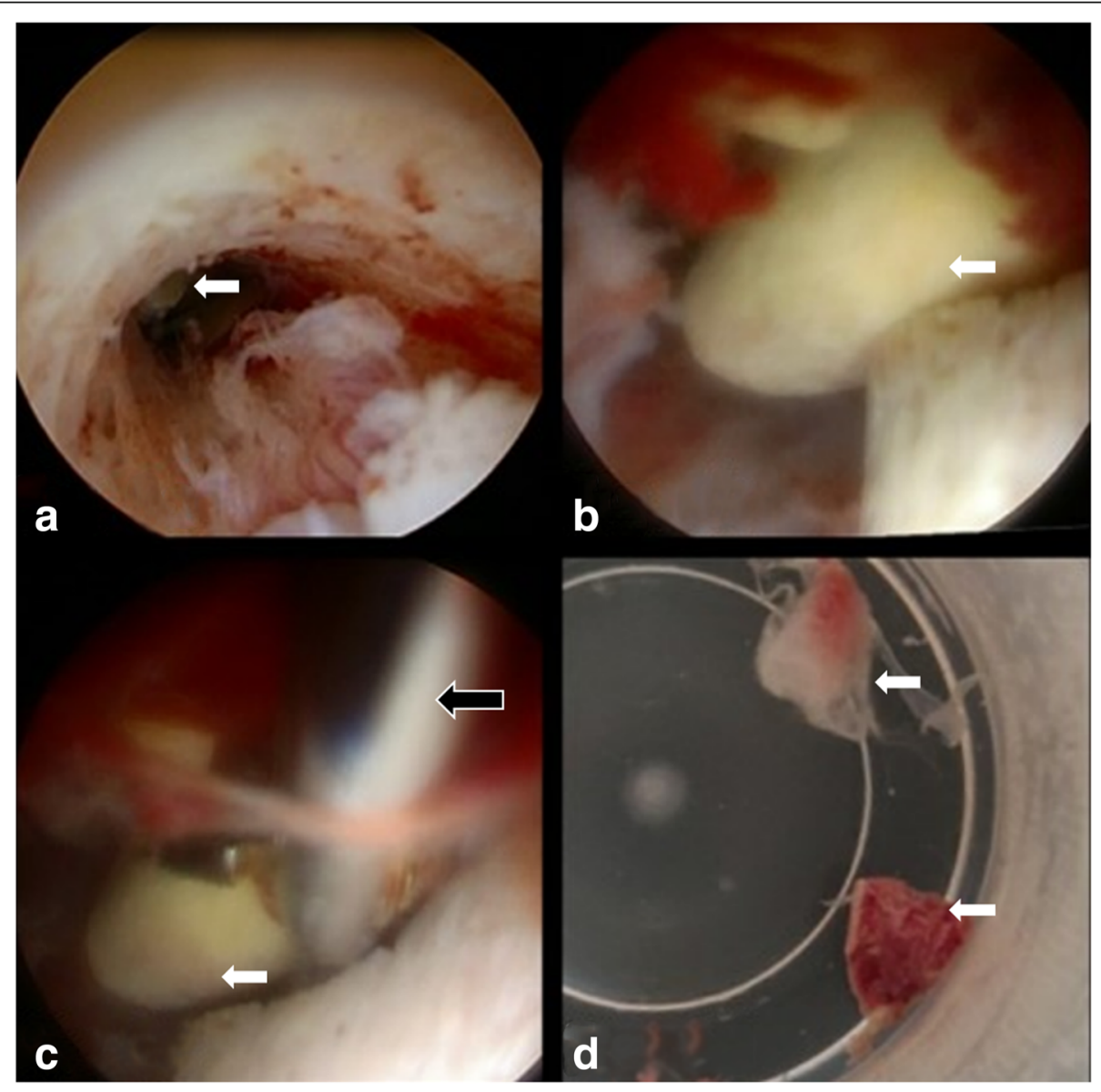

Fig. 6 Serial hysteroscopic images of Fig. 1 patient showing distant (a) and close-up (b) view of ossified (ivory bone) endometrial tissue (white block arrows). c Hysteroscopy biopsy forceps (black block arrow) within endometrial cavity during retrieval of bony endometrial bits (d) 


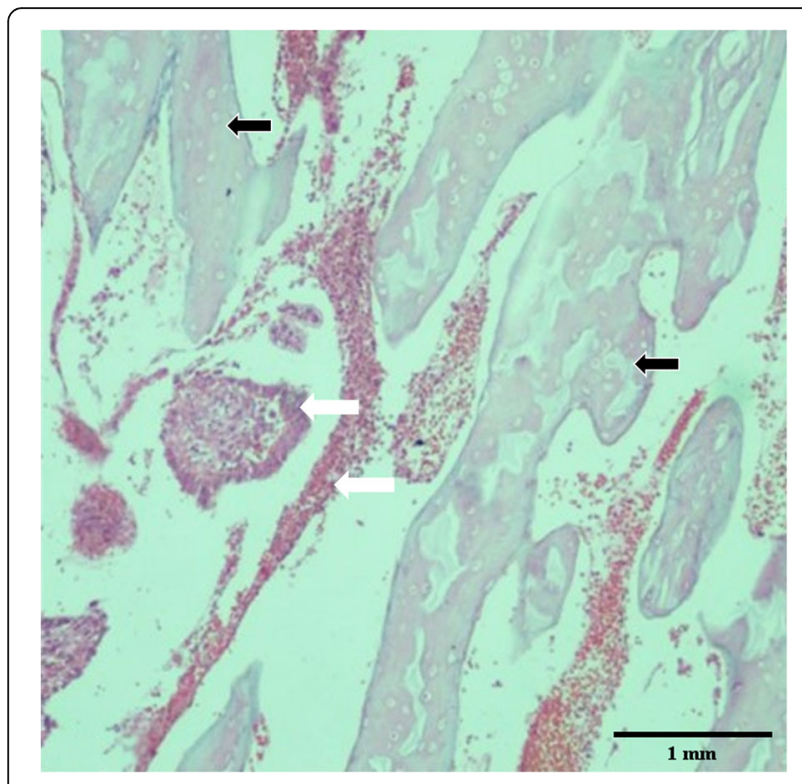

Fig. 7 Low power (10x) view photomicrograph showing endometrial glands and stroma (white block arrows) infiltrated by lamellar (black block arrows) bone (EOM). Endometrial stroma is also infiltrated by lymphocytes. Areas of haemorrhage are also seen

system concept was put forth. Superoxide dismutase system is a protective system and has a key role in endometrial differentiation. It is probable that post-abortion endometrial inflammation due to retained gestational tissues stimulates release of various factors like tumour necrosis factor from the mononuclear phagocytes. Deficient superoxide dismutase in endometrium may

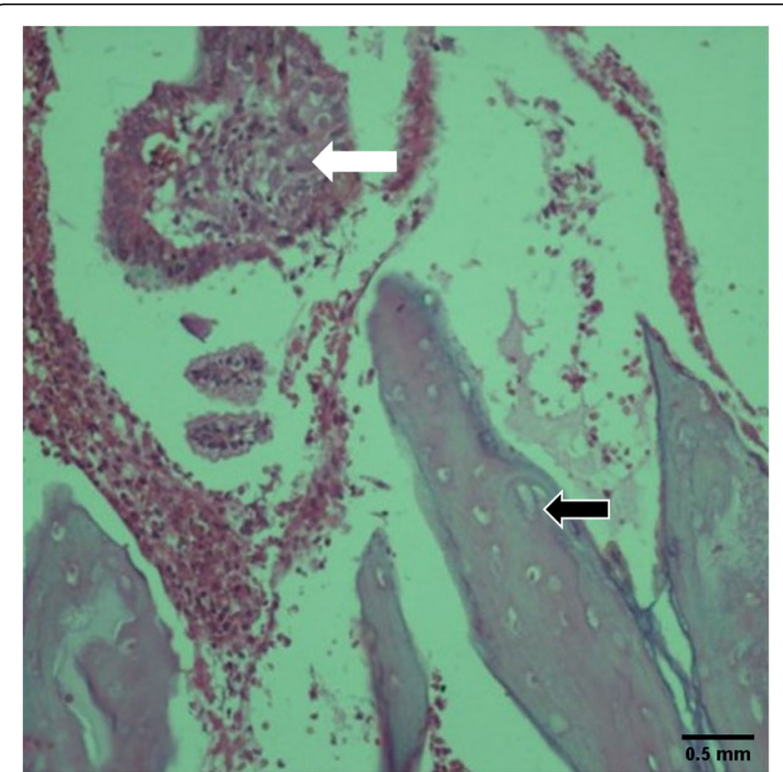

Fig. 8 High power (40x) view photomicrograph showing single endometrial gland (white block arrow) and stroma infiltrated by bony trabeculae (black block arrow). perhaps lead to chronic exposure of multipotential stromal cells to these inflammatory factors eventually leading to transformation of these endometrial stromal cells into osteoblasts [13]. The mean duration between postabortion instrumentation and detection of endometrial ossification in our study was $5.1 \pm 1.8$ years justifying the time required for metaplastic transformation. Six cases had first trimester abortion in our study and three had history of early second trimester abortion. It is also proposed that ossification of the foetal skeleton reaches a certain level in second trimester with foetal skeleton serving as a source of calcium for subsequent endometrial ossification [14]. Pathologically minimal adjacent endometrial reaction with endochondral ossification may help to distinguish osseous metaplasia from retained foetal osteoblastic tissue after an abortion [1, 14].

Three out of four patients in whom HPE was consistent with calcific endometritis did not have any history of abortion or instrumentation where as one patient had a previous second trimester abortion. Dystrophic calcification of retained and necrotic tissues may explain the endometrial calcification in genital tuberculosis with overlapping contribution of chronic endometritis [15]. Calcific endometritis is histologically distinct from EOM. Calcific endometritis shows abundant inflammatory cells and interspersed calcific foci. On the contrary, EOM has an endogenous development with metaplastic transformation of stromal cells into trabecular or lamellar bone [13]. However, on USG calcific endometritis is indistinguishable from EOM as both show linear or tubular echogenic endometrium with posterior acoustic shadowing. This may be confined to corpus uteri or may extend into endocervical canal. Endometrial tuberculosis causing endometrial calcification is sonographically indistinguishable from EOM; however, past history of pulmonary tuberculosis, presence of complex adnexal cystic lesions and pelvic ascites are pointers towards the diagnosis of endometrial tuberculosis. On histology abundance of chronic inflammatory granulomatous infiltrates with interspersed foci of calcification is characteristic of endometrial tuberculosis. Additionally, detection of AFB-specific nucleic acid in menstrual blood or vaginal discharge may be found in these cases.

Other common differential diagnosis of EOM on USG is intra-uterine contraceptive device but the history is usually forthcoming about its insertion. IUCD is usually discrete linear echogenic structure (Fig. 9) with separate wings in upper uterine body in axial plane which helps in discriminating between IUCD and EOM. Less common differential diagnosis includes retained foetal tissue. Rare differential diagnosis of EOM on USG includes uterine teratomas, mixed mesodermal tumours and malignant mixed Mullerian tumours. However, presence of mass like morphology with associated mass effect is a distinguishing feature. 


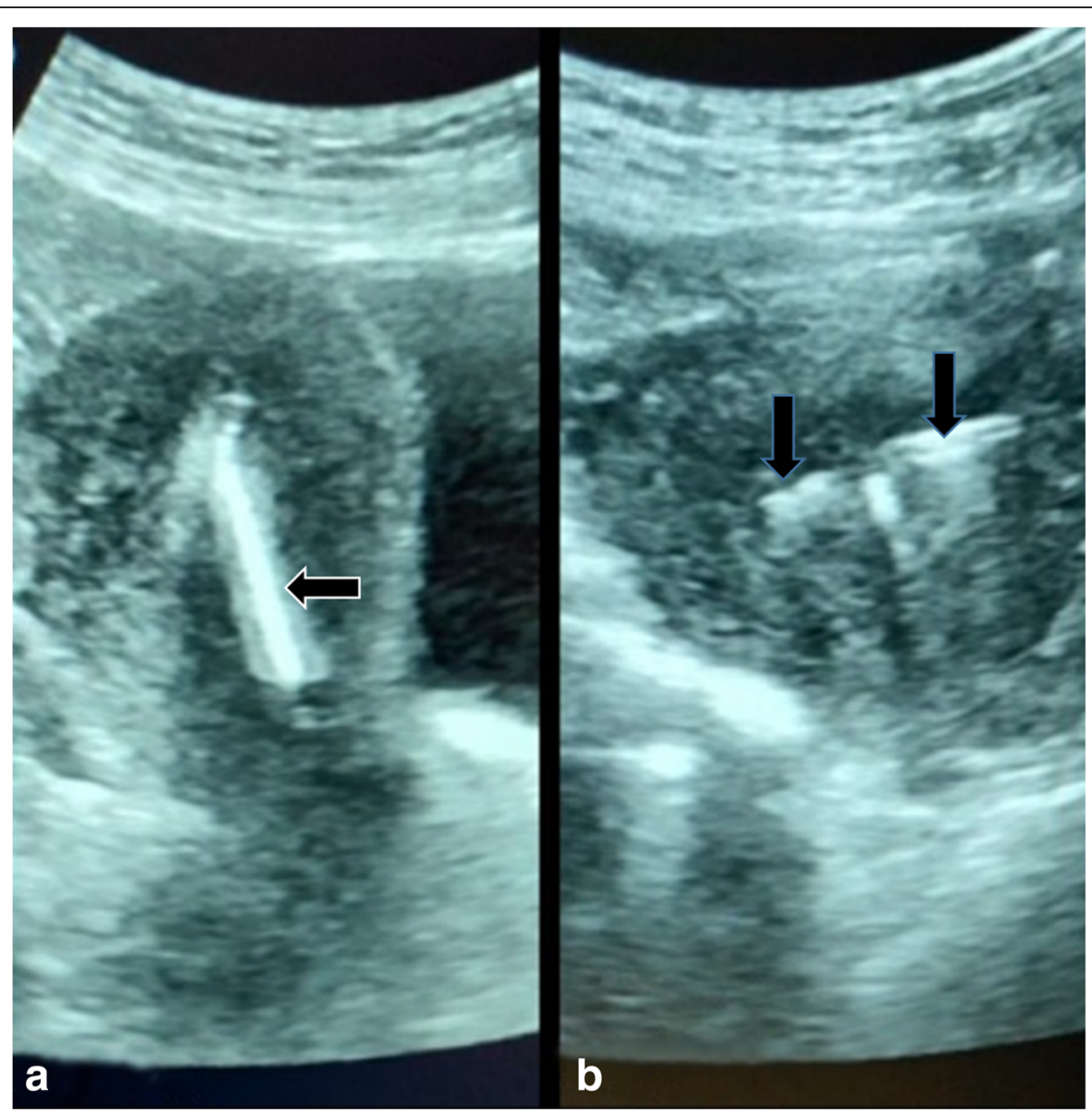

Fig. 9 Sagittal (a) and axial (b) transabdominal ultrasound images of intra-uterine Contraceptive device (black block arrows) mimicking endometrial ossification

MRI examination revealing loss of normal T2W hyperintense endometrial signal with T1W hypointense signal may serve as a complementary tool in establishing a diagnosis of EOM. However, MRI of pelvis is not warranted for the primary diagnosis of EOM in our view, as it has poor sensitivity and specificity for detecting ossification in addition to being expensive and selectively available [16]. However, MRI may be required in patients with uncertain or additional uterine (adenomyosis or mass) or adnexal (endometriosis or suspected genital tuberculosis) findings on USG. CT scan though having an extremely high sensitivity for detection of ossification is of limited value due to significant radiation exposure to the patient.

The exact prevalence of EOM is unknown. Based on a compendious review of literature spanning almost a century from 1928 to 2016, Khan SN et.al concluded that a total of 293 cases have been reported in literature [17]. Most of the knowledge about EOM has emanated from case reports and small case series. However, the wide use of USG has resulted in increased detection of this condition. In this review, they found that antecedent history of uterine instrumentation related to pregnancy termination was present in $88 \%$ patients. The compiled review concluded that infertility $(56.2 \%)$ was the most common presenting symptom followed by menstrual irregularities (19.8\%), vaginal discharge (6.4\%), pelvic pain (7.9\%) and dysmenorrhea (2.6\%). Some patients were asymptomatic and were found incidentally. The USG findings were more or less similar in the reported cases with presence of linear echogenic structure within endometrial canal. They also reported that a majority of patients conceived spontaneously after treatment.

Majority of reported cases of endometrial ossification have an antecedent history of pregnancy loss. But the reported cases do not elaborate whether endometrial ossification was a retained foetal bone or de novo bone formation. Cayuela $\mathrm{E}$ et al. attempted to unravel this mystery by performing genetic analysis of the retrieved endometrial bone and the woman, and concluded that the genetic markers of endometrial bone completely matched that of woman thus leading to the conclusion that endometrial bone is not retained foetal bone but true de novo osseous metaplasia [18]. 
Infertility is a common presentation in EOM as was seen in all patients in the present study. The relationship between infertility and EOM is not well established. However, reactive endometritis caused by metaplastic bone may interfere with blastocyst implantation [15]. Lewis et al. reported 50\% reduction in local prostaglandin concentration after therapeutic dilatation and curettage in these cases supporting inflammation as a contributing factor in causing infertility [17]. Another plausible explanation for associated infertility is osteoblastic bone acting as a foreign body, a mechanism similar to intra-uterine contraceptive device preventing conception $[12,18,19]$. Complete removal of this metaplastic tissue restores fertility $[12,18,19]$. Uterine curettage or hystercopically guided removal of endometrial ossification is the treatment of choice [20,21]. Recently, ultrasound-guided hysteroscopic removal of bone has been recommended due to its good visibility which helps in complete removal of the metaplastic tissue embedded even in adjacent myometrium [12, 22, 23]. Genital tuberculosis was likely the cause of endometrial calcification in four of our patients amongst which one had history of pulmonary tuberculosis. In endemic country like ours where genital tuberculosis is so common, it must be included in the diagnostic workup of endometrial calcification.

The present study is the largest study in terms of number of EOM patients reported. However, the singlecentre design is a limitation of our study. The present study may not reflect the actual incidence of EOM because the patients in our study were symptomatic for infertility and attended infertility clinic. As a result, it will miss those cases who are not infertile, who have other symptoms like pelvic pain, vaginal discharge or menstrual irregularity or who may not be attempting conception.

\section{Conclusion}

In nutshell, endometrial osseous metaplasia is a rare and treatable cause of secondary infertility with ultrasound being the best non-invasive diagnostic imaging modality. Post-abortion instrumentation is the most common aetiology; however, genital tuberculosis must be included in the diagnostic workup especially in endemic zones. The actual pathogenesis is debatable and area of active research. Ultrasound-guided hysteroscopic removal/dilatation and curettage is the treatment of choice and usually curative for infertility.

\section{Abbreviations}

ATT: Anti-tubercular treatment; CT: Computed tomography; EOM: Endometrial osseous metaplasia; HPE: Histopathologic examination; HSG: Hysterosalpingography; IUCD: Intra-uterine contraception device; MRI: Magnetic resonance imaging; S.D: Standard deviation; TAS: Transabdominal sonography; TVS: Trans-vaginal sonography; USG: Ultrasound

\section{Acknowledgements}

Acknowledgment of grants, disclosures or other assistance:

I, Wani Abdul Haseeb, have no disclosures regarding grants or other assistance.

I, Parry Arshed Hussain, have no disclosures regarding grants or other assistance.

I, Feroz Imza, have no disclosures regarding grants or other assistance. I, Jehangir Majid, have no disclosures regarding grants or other assistance. I, Rashid Masarat, have no disclosures regarding grants or other assistance.

\section{Authors' contributions}

WA, PA and JM performed, analysed and interpreted the ultrasound, computed tomography $(\mathrm{CT})$ and magnetic resonance images. Fl performed the histopathological examination of specimen and was also involved in obtaining hysteroscopic specimen. RM performed hysteroscopies, dilatation and curettage and provided lab, hormonal and biochemical parameters. All authors were involved in manuscript preparation and literature research. All the authors have read and approved the manuscript.

\section{Funding}

No funding was required for this study as it was the part of infertility evaluation as per the institutional protocol. The patients paid themselves the nominal fee for the procedure.

\section{Availability of data and materials}

All the data and materials were obtained from patients registered in our hospital.

\section{Ethics approval and consent to participate}

IRB statement: This study was duly approved by the Institutional Review Board (IRB). The study didn't require a reference number. No animal participants were used in this study. Informed verbal consent in the vernacular language was obtained from illiterate patients and written consent in patients who could read.

\section{Consent for publication}

None

\section{Competing interests}

We declare that we have no (financial and non-financial) competing interests.

\section{Author details}

'Department of Radiodiagnosis, Government Medical College, Srinagar, Jammu and Kashmir 190010, India. ²Department of Radiodiagnosis,

Sher-i-Kashmir Institute of Medical Sciences, Srinagar, Jammu and Kashmir 190010, India. ${ }^{3}$ Department of Pathology, Sher-i-Kashmir Institute of Medical Sciences, Srinagar, Jammu and Kashmir 190010, India. ${ }^{4}$ Department of Obstetrics and Gynecology, Directorate of Health Services, Srinagar, Jammu and Kashmir 190010, India.

Received: 15 May 2020 Accepted: 2 December 2020

Published online: 11 December 2020

\section{References}

1. Garg D, Bekker G, Akselrod F. Endometrial osseous metaplasia: an unusual cause of infertility. BMJ Case Rep. 2015;2015:bcr2015209523.

2. Ceccacci L, Clancy G (1981) Endometrial ossification: report of an additional case. Am J Obstet Gynecol 141:103-104

3. Acharya U, Pinion SB, Parkin DE, Hamilton MPR (1993) Osseous metaplasia of the endometrium treated by hysteroscopic resection. Br J Obstet Gynaecol 100:391-392

4. AbdullGaffar B, AlMulla A (2020 Mar) Endometrial calcifications. Int J Surg Pathol 1:1066896920909425

5. Jamal I, Gupta RK, Raj S, Bhadani PP (2020) Endometrial and cervical osseous metaplasia with extramedullary haematopoiesis presenting as amenorrhea: Report of three cases. Indian J Pathol Microbiol 63(2):295

6. Rodriguez BD, Adamson GD (1993) Hysteroscopic treatment of ectopic intrauterine bone A case report. J Reprod Med 38:515-520 
7. Guerra LF, Pessanha LB, Oliveira GA, Melo AM, Braga FS, Souza RS (2016 Feb) Endometrial osseous metaplasia: sonographic, radiological and histopathological findings. Radiol Bras 49(1):62-63

8. DL SJ (2020) Endometrial Osseous metaplasia associated with secondary infertility-Report of 2 cases. University Journal of Surgery and Surgical Specialities 31:6(1)

9. Herbold DR, Magrane DM (1986) Calcifications of the benign endometrium. Arch Pathol Lab Med 10:666

10. Lainas T, Zorzovilis I, Petsas G, Alexopoulou E, Lainas G (2004) loakimidis T: Osseous metaplasia: case report and review. FertilSteril 82:1433-1435

11. Sood A, Lewis E, Grey B, Mohiyiddeen L (2019) Novel management of endometrial osseous metaplasia. World J Surg Surgical Res 2:1164

12. Bahceci M, Demirel LC (1996) Osseous metaplasia of the endometrium: a rare cause of infertility and its hysteroscopic management. Hum Reprod 11: 2537-2539

13. Rosa-e-Silva JC, Barcelos ID, Navarro PA, de Sá Rosa AC, Nogueira AA, Ferriani RA (2009 Dec) Osseous metaplasia of the endometrium associated with infertility: a case report and review of the literature. J Med Case Rep 3(1):1-4

14. Perino A, Mangione D, Svelato A, Forlani F, Gargano F, Incandela D, Coppola MA, Venezia R (2013 Jan) Chronic renal failure and endometrial osseous metaplasia: a hypothetical pathway. Acta Obstet Gynecol Scand 92(1):118-119

15. Tyagi SP, Saxena K, Rizvi R, Langley FA (1979) Foetal remnants in theuterus and their relation to other uterine heterotopia. Histopathology. 3(4):339-345

16. Marcus SF, Bhattacharya J, Williams G, Brinsden P. Hamou J (1994) Endometrial ossification: a case of secondary infertility. Report of two cases. Am J ObstetGynecol 170:1381-1383

17. Khan SN, Modi M, Hoyos LR, Imudia AN, Awonuga AO (2016 Jul) Bone in the endometrium: A review. International journal of fertility \& sterility 10(2):154

18. Cayuela E, Perez-Medina T, Vilanova J, Alejo M, Cañadas P (2009 Apr 1) True osseous metaplasia of the endometrium: the bone is not from a fetus. Fertil Steril 91(4):1293-12e1

19. Lewis V, Khan-Dawood F, King M, Beckham C (1990) Dawood MY:Retention of intrauterine fetal bone increases menstrual prostaglandins. ObstetGynecol 75:561-563

20. Chotaliya AB, Saifi SG, Achuthan G (2018) Sonographic appearance of endometrial osseous metaplasia. J Clin Ultrasound 46(8):536-539

21. Umashankar T, Patted S, Handigund RS (2010) Endometrial osseous metaplasia: Clinicopathological study of a case and literature review. Journal of human reproductive sciences 3(2):102

22. Madaan M, Suman S, Sharma R, Kapoor N, Garg P, Raj SS (2015) Osseous metaplasia of the endometrium and successful hysteroscopic resection: a report of two cases and a review of the literature. Asian J Endosc Surg 8(1): 63-66

23. Roach MK, Thomassee MS (2015) An incidental finding of endometrial osseous metaplasia during office hysteroscopy. Am J Obstet Gynecol 212(3): 402-4e1

\section{Publisher's Note}

Springer Nature remains neutral with regard to jurisdictional claims in published maps and institutional affiliations.

\section{Submit your manuscript to a SpringerOpen ${ }^{\circ}$ journal and benefit from:}

- Convenient online submission

- Rigorous peer review

- Open access: articles freely available online

- High visibility within the field

- Retaining the copyright to your article

Submit your next manuscript at $\boldsymbol{\nabla}$ springeropen.com 